\title{
聚合物对单壁碳纳米管增溶及选择性分离的机理研究进展
}

\author{
杨阳 ${ }^{a, b}$ 李若梅 ${ }^{b, c}$ 王 炜 ${ }^{d}$ 许子文 $^{b}$ 谢光辉 ${ }^{d}$ \\ 鲁郑全 $d$ 李靖靖 $d$ 宋力平 $*, a$ 李维实 ${ }^{*}, b, d$ \\ $\left({ }^{a}\right.$ 上海大学理学院 上海 200444) \\ ( ${ }^{b}$ 中国科学院上海有机化学研究所 中国科学院有机功能分子合成与组装化学重点实验室 上海 200032) \\ ( ${ }^{c}$ 上海师范大学化学与材料科学学院 上海 200234) \\ ( ${ }^{d}$ 郑州工程技术学院高性能有机功能材料工程中心 郑州 450044)
}

\begin{abstract}
摘要 现有方法制备的单壁碳纳米管，混杂着半导体性和金属性两种碳管，需分离纯化后才能发挥这两种碳管各自的 优异性能和广泛应用. 在众多已开发的方法中, 利用聚合物非共价相互作用实现半导体型和金属型碳管的选择性分离 被认为是操作最简单、效率最高、无损碳管结构性能的方法. 迄今为止, 大量相关工作已被报道, 开发了众多的聚合物 体系, 提出了各种分离纯化机理, 但目前仍然没有统一的认识. 综述了近年来有关单壁碳纳米管增溶和选择性分离的 研究进展, 分析了聚合物与单壁碳纳米管之间的相互作用, 探讨了聚合物结构、分子量、侧链长度、聚合物与碳管比 例、温度、溶剂等因素对碳管增溶与选择性分离的影响, 比较了各种作用机理, 并提出了自己的观点.
\end{abstract}

关键词＼cjkstart单壁碳纳米管; 聚合物; 选择性分离; 非共价相互作用; 作用机理

\section{Research Advances on the Mechanism of Polymer Solubilization and Selective Separation of Single-Wall Carbon Nanotubes}

\author{
Yang, Yang ${ }^{a, b}$ \\ Li, Ruomei ${ }^{b, c}$ \\ Wang, Wei ${ }^{d}$ \\ $\mathrm{Xu}, \mathrm{Zi}-\mathrm{Wen}^{b}$ \\ Xie, Guanghui ${ }^{d}$ \\ Lu, Zhengquan ${ }^{d}$ \\ Li, Jingjing ${ }^{d}$ \\ Song, Liping*,a \\ Li, Wei-Shi*,b,d \\ ( ${ }^{a}$ College of Science, Shanghai University, Shanghai 200444) \\ $\left({ }^{b}\right.$ Key Laboratory of Synthetic and Self-Assembly Chemistry for Organic Functional Molecules, Shanghai Institute of \\ Organic Chemistry, Chinese Academy of Sciences, Shanghai 200032) \\ ( ${ }^{c}$ College of Chemistry and Material Sciences, Shanghai Normal University, Shanghai 200034) \\ ( ${ }^{d}$ Engineering Research Center of Zhengzhou for High Performance Organic Functional Materials, \\ Zhengzhou Institute of Technology, Zhengzhou 450044)
}

\begin{abstract}
Single-walled carbon nanotubes (SWNTs) prepared by present methods are a mixture of semiconducting and metallic ones, which need to be separated and purified to give them full play with their excellent properties and for their attractive applications. Among numerous developed methods, the selective separation of semiconducting and metallic SWNTs by polymer non-covalent interactions is considered to be the most simple, efficient and no-damage to carbon nanotube structure and properties method. So far, a lot of works have been reported, and many polymer systems have been developed. Meanwhile, various separation and purification mechanisms have been proposed, but there are still no unified and convincing understandings. In this review, the reported SWNT solubilization and selective separation works are summarized, the interactions between polymer and SWNTs are analyzed, and the effects of polymer structures, molecular weights, side chain lengths, polymer/SMNT ratio, temperature, and solvent are focally discussed. After comparing various separating mechanisms, our own understandings and views are put forward.
\end{abstract}

Keywords single-wall carbon nanotubes; polymer; selective separation; non-covalent interactions; mechanisms

\footnotetext{
* Corresponding authors. E-mail: lpsong@shu.edu.cn; liws@mail.sioc.ac.cn

Received June 11, 2020; revised July 29, 2020; published online August 11, 2020

Dedicated to Professor Henry N. C. Wong on the occasion of his 70th birthday.

Project supported by the National Natural Science Foundation of China (Nos. 21674125, 21672251, 51761145043), the Strategic Priority Research Program of Chinese Academy of Sciences (No. XDB20020000) and the Zhengzhou Institute of Technology.

国家自然科学基金(Nos. 21674125, 21672251, 51761145043)、中国科学院先导计划(No. XDB20020000)和郑州工程技术学院资助项目.
} 
碳纳米管是 1991 年由 Iijima ${ }^{[1]}$ 在高分辨率显微镜下 发现的一种由石墨烯卷曲而成的管状一维结构纳米材 料. 单壁碳纳米管(下文简称碳管), 由单层石墨烯卷曲 而成, 其结构是由碳管垂直横截面剪开铺展并在石墨烯 晶格上对齐芳环结构后横截面圆周所形成的矢量 $\boldsymbol{C}_{\mathbf{h}}$ 表 示. 由于 $C_{\mathbf{h}}$ 与石墨烯晶格上单胞基础矢量 $\boldsymbol{a}_{1} 、 \boldsymbol{a}_{\mathbf{2}}$ (石墨 烯晶格单胞为苯环结构, $\boldsymbol{a}_{1}$ 矢量是从苯环 1 位指向 3 位, 而 $\boldsymbol{a}_{2}$ 则是从苯环 1 位指向 5 位)之间的关系为: $\boldsymbol{C}_{\mathrm{h}}=n \boldsymbol{a}_{1}$ $+m \boldsymbol{a}_{2}, n 、 m$ 为整数, 则该碳管用 $(n, m)$ 表示其结构, 称 之为手性指数. 图 1a 展示了 $(6,6)$ 碳管的结构示意图 ${ }^{[2]}$. 当 $|n-m|$ 为 3 的倍数时, 此种 $(n, m)$ 碳管为金属型碳 管; 反之, 当 $|n-m|$ 不能被 3 整除时, 此种 $(n, m)$ 碳管 为半导体型碳管. 碳管结构的另外一种表达方式, 由手 性角 $\theta$ 和碳管直径 $h$ 构成(图 $1 \mathrm{~b}$ ). 根据手性角的不同, 碳 管分为扶手型、锯齿型和其他手性(图 1c $)^{[3]}$. 在研究聚 合物对碳管的选择时, 碳管的手性角和直径是关键的两 个参数.

碳管在半导体器件领域的应用发展非常迅速 ${ }^{[4-6]}$. 但是在制备碳管的过程中，目前还没办法实现半导体型 和金属型碳管的选择性制备, 所以对碳管的分离纯化成 为很多碳管应用领域研究的重点. 近年来发展了很多分 离方法, 尤其是能保留碳管侧壁完整结构的、不破坏其 电性的方法, 包括利用混合碳管中不同结构碳管密度的 差异实现分离的密度梯度离心法 ${ }^{[7]}$ 、利用不同电性碳管 在电场中的不同表现来分离的电泳法 ${ }^{[8]}$ 、利用有机物与 碳管非共价相互作用分离的 DNA 包裹法 ${ }^{[9]}$ 和聚合物缠 绕法 ${ }^{[10-12]}$. 这些方法对半导体型和金属型碳管的分离各 有千秋, 本综述主要关注聚合物缠绕法, 通过总结聚合 物与碳管通过非共价相互作用实现增溶和选择性增溶 的工作, 探讨了聚合物结构、侧链长度、温度、聚合物
分子量和聚合物与碳管比例、溶剂的影响, 试图理解聚 合物对半导体型碳管和金属型碳管选择性分离的机制.

\section{1 聚合物对碳管的增溶}

聚合物对碳管的增溶是指聚合物通过与碳管相互 作用，使得单个碳管分散在溶剂中，而不是聚集成束. 增溶能力越强是指聚合物把碳管在溶剂中分散成单个 碳管的数量越多. 反之, 增溶能力越差是指聚合物不能 把碳管在溶剂中分散成单个碳管, 而使碳管在溶剂中以 束的形态存在, 或者直接聚集沉淀, 不能分散于溶剂中. 通过紫外-可见一近红外吸收光谱可以表征碳管在溶剂 中分散的形态. 如图 2a 所示, 相比于曲线 A、B、C, 曲 线 $\mathrm{D}$ 的峰更宽, 不尖锐, 这是因为碳管多数以束聚集态 分散在溶剂中, 使吸收光谱的峰以重叠的状态呈现, 而 曲线 A、B、C 的尖锐峰则是单个碳管呈现的吸收峰 ${ }^{[13]}$. Itkis 等 ${ }^{[14]}$ 进一步探讨了紫外-可见-近红外吸收谱图上 吸收峰的组成, 认为 S22 谱带是由碳管吸收、碳管等离 子吸收和非碳管碳杂质等离子吸收三部分组成, 通过碳 管吸收面积 $A A(S)$ 和 S22 谱带整体吸收面积 AA(T) 可计 算碳管的纯度 $R P$, 计算式为 $R P=[\mathrm{AA}(\mathrm{S}) / \mathrm{AA}(\mathrm{T})] /$ 0.141(图 2b). 至此, 增溶能力的强弱不仅能通过吸收光 谱峰的强度和尖锐度来表征，还能通过吸收峰定量计算 碳管纯度，并直观地对比.

增溶是碳管对聚合物最初的诉求. 在最初把碳管应 用于器件制备时发现，碳管很容易成束聚集，此时在制 备过程中加入聚乙烯会阻止碳管聚集，但由于多数都不 溶于溶剂中, 所以聚乙烯并不是把碳管分散到溶剂中的 优良分散剂. 在此之后, Shaffer 等 ${ }^{[15]}$ 发现聚乙烯醇与碳 管能进行非共价相互作用，实现增溶. 从此以后，碳管 的分散剂从聚乙烯、聚环氧乙烯和聚乙烯醇这类烷基 (a)

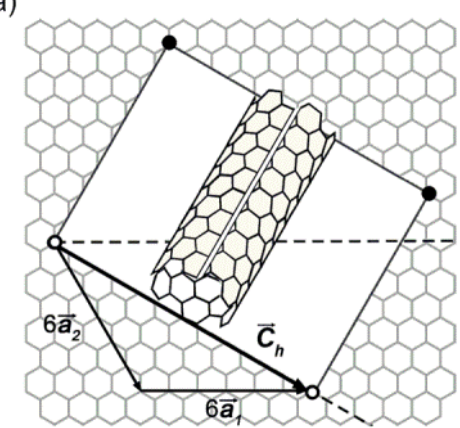

(b)

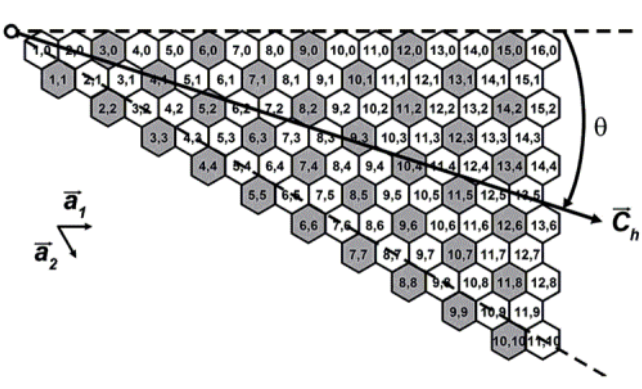

(c)

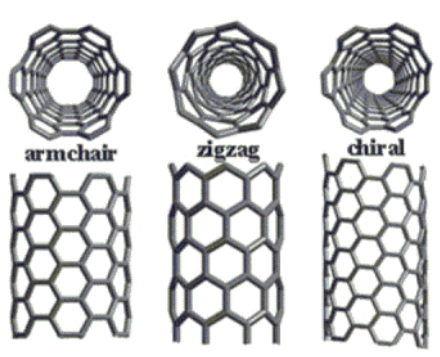

图 1 (a)以 $(6,6)$ 碳管为例示意从石墨烯片构造碳纳米管的图 ${ }^{[2]}$ (b)石墨烯层上可能出现的碳管 $(n, m)$ 结构和(c)从左到右为扶手型、 锯齿形和其他手性碳管结构图 ${ }^{[3]}$

Figure 1 (a) Illustration of the construction of a carbon nanotube from a graphene sheet using $(6,6)$ nanotube as an example; ${ }^{[2]}$ (b) the possible SWNT $(n, m)$ structures represented on a single graphene sheet: ${ }^{[2]}(\mathrm{c})$ the structures of armchair, zigzag and chiral nanotubes ${ }^{[3]}$ (a) The detail construction is to roll up the white area graphene sheet and overlap the corresponding two sets of lattice points. (b) Semiconducting SWNTs are white and metallic ones are shaded ${ }^{[2]}$. Reprinted with permission from Ref. [2]. Copyright 2008 American Chemical Society. (c) Reprinted with permission from Ref. [3]. Copyright 2011 Royal Society of Chemistry 

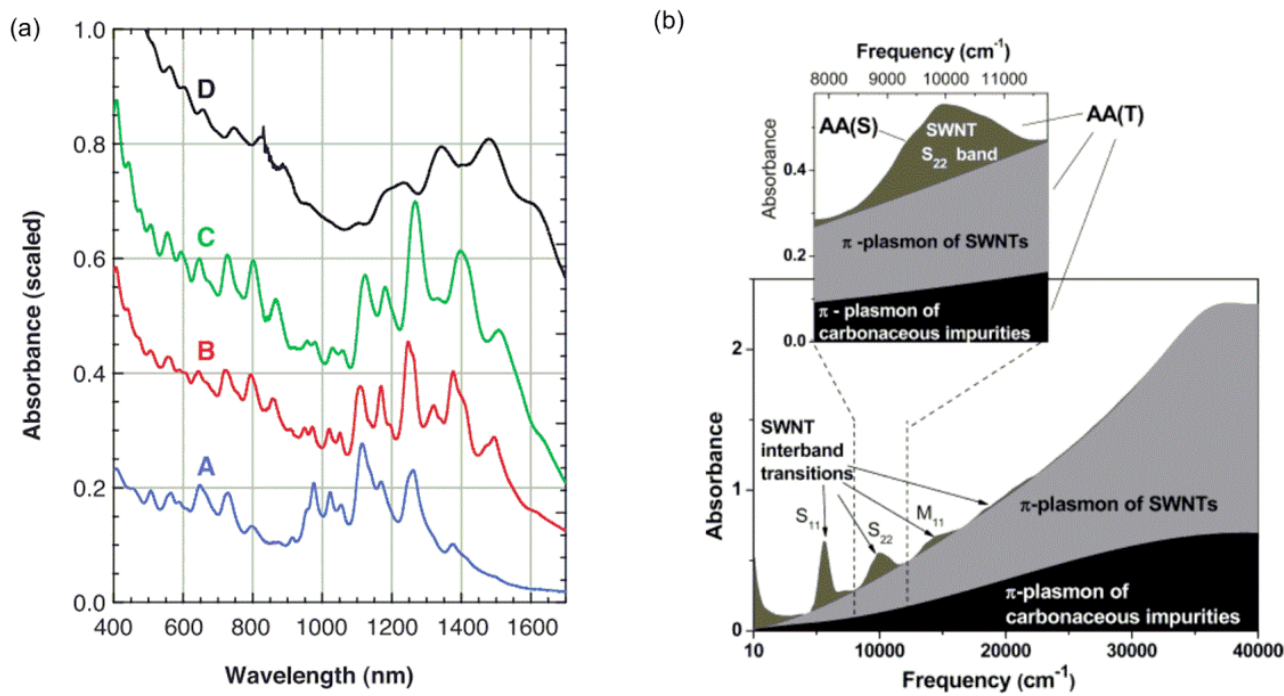

图 2 (a)曲线 A、B、C 的分散成单碳管的吸收光谱图和曲线 D 的聚集成小束碳管的吸收光谱图 ${ }^{[13]}$; (b)碳管的吸收光谱示意图 ${ }^{[14]}$ Figure 2 (a) Absorption spectra of curves A, B and C dispersed individual carbon nanotubes and absorption spectrum of curve D aggregated small bundle carbon nanotubes; ${ }^{[13]}$ (b) Electronic spectrum of a typical SWNT sample

(a) Reprinted with permission from Ref. [13]. Copyright 2002 the American Association for the Advancement of Science; (b) the inset shows the region of the S22 interband transition utilized for NIR purity evaluation. In the diagram, AA(S) area of the S22 spectral band after linear baseline correction and $\mathrm{AA}(\mathrm{T})$ total area of the S22 band including SWNT and carbonaceous impurity contributions. The NIR carbon tube relative purity is given by RP= (AA(S)/AA(T))/0.141 ${ }^{[14]}$. Reprinted with permission from Ref. [14]. Copyright 2005 American Chemical Society

结构的聚合物到聚苯撑乙烯、聚苯撑乙炔这类芳香结构 的聚合物, 再到聚噻吩和聚苆这类有选择性的芳香结构 的聚合物. 从这个过程中逐步找到了与碳管相互作用更 强的聚合物, 也就是增溶能力更强的聚合物. 更重要的 是, 实现了从无差别增溶到对金属型和半导体型碳管的 选择性增溶的全过程.

选择性增溶是指聚合物能在溶剂中选择性分散特 定直径、手性或导电性的碳管. 本综述主要探讨的是聚 合物对不同导电性碳管的选择性增溶. 聚合物对碳管的 选择性增溶是一种竞争性的增溶, 也就是聚合物在特定 环境下能对半导体型碳管和金属型碳管的增溶效果恰 好有明显差异, 使得半导体型碳管能在溶剂中分散更 多, 金属型碳管多数都聚集沉降, 从而利用聚合物选择 性增溶的能力制备出纯度更高的半导体型碳管. 半导体 型碳管和金属型碳管可以通过紫外-可见-近红外吸收 光谱表征, 图 $3 \mathrm{a}$ 是直径较小的碳管 $\mathrm{HiPCO}(0.8 \sim 1.2$ $\mathrm{nm})$ 的吸收光谱, 半导体型碳管和金属型碳管在不同的 吸收峰区域，黑线框标注区域(波长为 $450 \sim 600 \mathrm{~nm}$ ) 为 金属型碳管特征吸收峰区域 ${ }^{[16]}$. 图 $3 \mathrm{~b}$ 是直径较大的碳 管 SO (arc plasma jet) $(1.4 \mathrm{~nm})$ 的吸收光谱. 相比直径小 的碳管, 无论是半导体型还是金属型碳管的吸收波长都 更长, 但同样有明确的吸收峰区域, 黑线框标注区域 (波长为 $600 \sim 800 \mathrm{~nm}$ ) 为金属型碳管特征吸收峰区域. 聚合物对金属型碳管和半导体型碳管的选择性增溶使 得碳管在半导体器件领域的应用有更广阔的前景. 自从 聚苆被报道出具有选择性分离金属型碳管和半导体型
碳管的能力 ${ }^{[10]}$, 大量的聚芴衍生物被设计合成，同时也 有很多研究者试图发现新的能选择性分离金属型和半 导体型碳管的聚合物骨架结构. 迄今为止, 从聚合物骨 架结构层面总结，能实现选择性分离金属型碳管和半导 体型碳管的聚合物只有聚芴 ${ }^{[10]}$ 、聚噻吩 ${ }^{[11]}$ 和聚咔唑 ${ }^{[12]}$, 骨架结构是讨论聚合物对碳管选择性分离的机理时最 重要的部分 ${ }^{[17-18]}$.

\section{2 聚合物骨架结构}

对于实现碳管的增溶，最重要的是找到能与碳管相 互作用并有良好溶解度的试剂. 最早表面活性剂被用于 增溶碳管 ${ }^{[19-20]}$, 之后, 聚乙烯醇等具有两性的柔性聚合 物成了重要研究对象 ${ }^{[15]}$. 而后发现含芳香刚性骨架的 聚合物，除了能提供类似表面活性剂和柔性聚合物分子 类似的以范德华力为主的非共价相互作用之外，还能提 供更强的 $\pi-\pi$ 相互作用，成为目前非共价实现碳管增溶 的最主要方式.

\section{1 烷基主链的柔性聚合物}

早期认为聚乙烯醇之所以能对碳管实现增溶, 主要 依靠的原理是空间稳定理论 ${ }^{[21]}$, 认为聚乙烯醇对碳管 的分散并不是通过形成胶束来实现的，而是聚合物的疏 水端通过基本动力学驱动吸附在碳管上, 亲水端在水中 延伸膨胀，实现碳管的空间稳定. 同时期，聚甲基丙烯 酸甲酯(PMMA) $)^{[22]}$ 和十二烷基磺酸钠(SDS $)^{[13]}$ 等也都被 发现有增溶碳管的作用，但基本认为其原理是空间稳定 


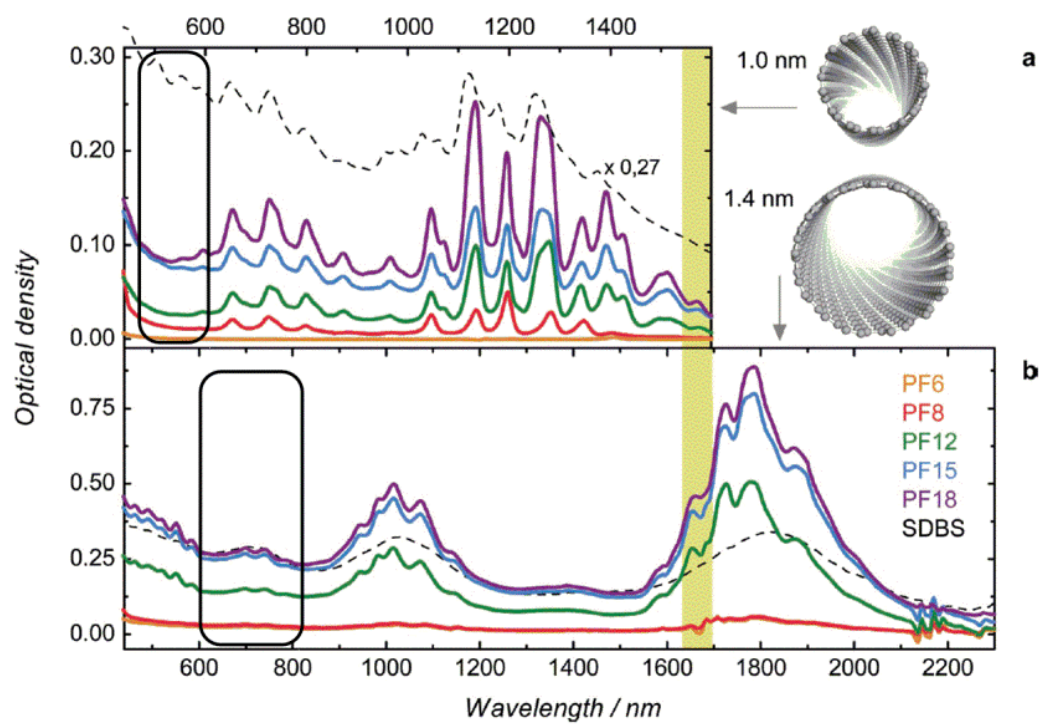

图 3 (a)聚合物选择性增溶直径为 $1 \mathrm{~nm}$ 碳管的吸收光谱; (b)聚合物选择性增溶直径为 $1.4 \mathrm{~nm}$ 碳管的吸收光谱(黑色框区域是金属 型碳管的特征吸收峰区域 ${ }^{[16]}$ )

Figure 3 (a) Absorption spectra of HiPCO SWCNTs (average diameter $1 \mathrm{~nm}$ ) dispersed with polymers; (b) absorption spectra of SO SWCNTs (average diameter $1.4 \mathrm{~nm}$ ) dispersed with polymers ${ }^{[16]}$

The black rectangle area is the typical absorption peak area of the metallic SWCNTs. Reprinted with permission from Ref. [16]. Copyright 2013 John Wiley and Sons

理论. 而后来 Crescenzo 等 ${ }^{[23]}$ 报道通过比较乙二醇自聚 的聚合物和乙二醇与丙烯硫化物共聚的聚合物对碳管 的包裹能力, 发现并不是如空间稳定理论预测的那样, 没有观察到亲水链段在水中的延伸和膨胀, 发现碳管表 面的覆盖率比理论上聚合物的覆盖率要高, 不能排除水 中的亲水链段悬空是因为碳管的表面积不够导致的. Yurekli 等 ${ }^{[24]}$ 报道了 SDS 在碳管表面形成无规则吸附层 (图 4c), 不同于过去认为的利用表面活性剂疏水端形成 圆柱形胶束(图 4a), 或者在碳管表面吸附成半胶束状 (图 4b)这两种机理. 并且 Didenko 等 ${ }^{[25]}$ 报道了利用苂光 的聚乙烯吡咯烷酮分散碳管, 通过电子显微镜观察到聚 合物能通过包裹在碳管表面分离出单个碳管, Manivannan 等 ${ }^{[26]}$ 报道了聚乙烯吡咯烷酮能把碳管很好的分散到 水和多种有机溶剂中, 这说明烷基链结构的聚合物能通 过包裹在碳管表面形成复合物, 起到分散剂的作用. 通 过总结报道的较多的几种以烷基为主链的聚合物对碳 管的增溶研究, 发现作为表面活性剂引入的聚合物, 并 非按照亲水和疏水的原理实现增溶, 而是与碳管形成了 非共价相互作用, 覆盖在碳管表面, 实现对碳管的增溶. 并且分子动力学模拟也研究了这种柔性骨架聚合物在 碳管表面覆盖的方式 ${ }^{[27]}$, 发现聚合物对碳管的包裹并 非通过确定的构型或规则的缠绕方式, 不同的聚合物与 碳管相互作用的构型受聚合物侧链影响很大. 前面提到 侧链是芳香基团的聚乙烯吡咯烷酩 ${ }^{[25]}$ 就能通过规则的 缠绕方式包裹碳管, 而大部分柔性骨架聚合物是无法实 现的. 总之, 柔性骨架的聚合物与碳管之间有相互作用, (a)
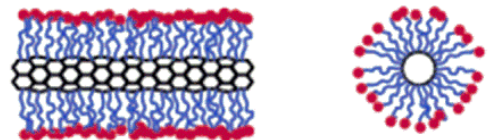

(b)

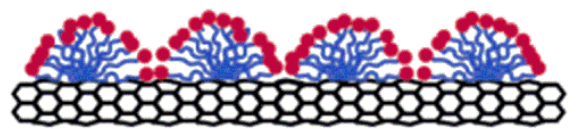

(c)

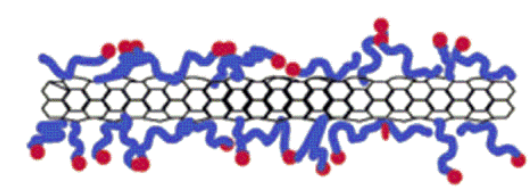

图 4 表面活性剂分子在碳管表面形成的(a)胶束(左：侧面; 右: 横切面)、(b)半胶束排列和(c)不规则吸附排列 ${ }^{[24]}$

Figure 4 (a) SWNT encapsulated in a cylindrical surfactant micelle (left: side view, right: cross section); (b) Hemimicellar adsorption of surfactant molecules on a SWNT; (c) Random adsorption of surfactant molecules on a SWNT ${ }^{[24]}$

Reprinted with permission from Ref. [24]. Copyright 2004 American Chemical Society

但聚合物覆盖在碳管表面的构型并不确定，并且通过后 面跟刚性骨架的聚合物对比发现, 柔性骨架的聚合物与 碳管的相互作用很微弱，导致其无法规则的覆盖在碳管 表面，虽然能实现增溶，但相比于刚性骨架聚合物，增 溶效率不高，也没有选择性.

\section{2 苯环主链的刚性聚合物}

芳香结构中最常用的就是苯环，而以苯环或苯环的 
衍生物为骨架的聚合物自然成了研究最多的结构. 能对 碳管实现增溶的以苯环为主链的刚性聚合物结构中, 聚 苯撑乙烯、聚苯撑乙炔、聚芴和聚咔唑是研究最多的几 种骨架结构(图 5).

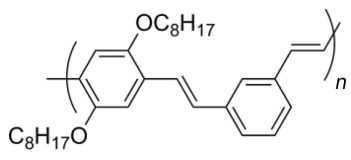

Poly(m-phenylene-co-2,5dioctoxy-p-phenylenevinylene) (PmPV)

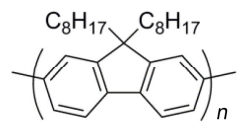

Poly $(9,9$ '-dioctyl fluorene $)$ (PFO)

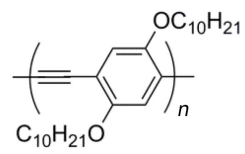

Poly(arylene ethynylene) (PAE)

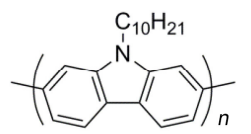

Poly(9-decyl carbazole)

(PC)
图 5 以苯环为主构筑单元刚性聚合物例子

Figure 5 Examples of rigid polymers based on benzene unit

1998 年, Curran 等 ${ }^{[28]}$ 发现 poly ( $m$-phenylene-co-2,5dioctoxy-p-phenylenevinylene) (PmPV)能和多壁碳管形 成复合物, 证明了两者之间的相互作用, 并提出主要是 $\pi-\pi$ 相互作用使得 PmPV 缠绕在碳管表面. 随后, 他们 通过 $\mathrm{PmPV}$ 与单壁碳纳米管的作用实现对单壁碳纳米 管的增溶, 并且通过 PmPV 与聚苯乙烯的对比证明了 PmPV 对碳管的增溶并非通过粘度或者浮力效应 ${ }^{[29]}$. Dalton 等 ${ }^{[30}$ 通过电子显微镜观察到 PmPV 与碳管相互作 用后碳管束被破坏, 制备出游离的单个碳管, 同时通过 拉曼光谱发现 PmPV 对特定直径或者特定直径范围的 碳管有选择性. Yi 等 ${ }^{[31]}$ 报道顺式的 PmPV 对碳管的增溶 效率是反式的二倍. 通过聚合物 PmPV 构型对碳管增溶 能力的影响结果, 可以判断聚合物在碳管表面的覆盖并 非如柔性聚合物那样是无规则的, 并且 Panhuis 等 ${ }^{[32]}$ 通 过分子动力学模拟也证明了 PmPV 与碳管相互作用时, 最适配的构型是缠绕在碳管表面. $\mathrm{Yi}$ 等 ${ }^{[33]}$ 报道了 $\mathrm{PmPV}$ 对直径为 $1.1 \sim 1.3 \mathrm{~nm}$ 的碳管增溶效率更高, 证实了 PmPV 对碳管直径有一定的选择性. 这些工作表明了 $\mathrm{PmPV}$ 与碳管之间的 $\pi-\pi$ 相互作用相当强, 导致聚合物 构型对增溶效果影响很大. 但是 PmPV 对金属型和半导 体型碳管之间的分离作用, 文献并没有给出确切的结 果.

聚苯撑乙炔相比于 PmPV 更富电子, 而且是直线型 结构聚合物. Chen 等 ${ }^{[34}$ 报道了 poly(aryleneethynylene)s (PAE) 能通过 $\pi-\pi$ 相互作用实现对碳管的增 溶, 效果明显. 不同于 PmPV 在碳管表面缠绕的方式, PAE 是以平行于碳管的方式吸附在碳管表面(图 6). 所 以相比于 PmPV, PAE 对直径较小的碳管增溶效果较好,

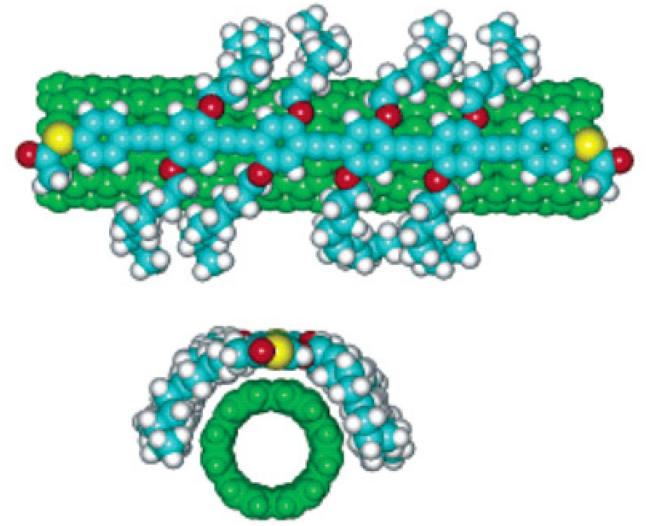

图 6 PAE 与碳管作用示意图 ${ }^{[34]}$

Figure 6 Model of PAE-SWNTs ${ }^{[34]}$

Reprinted with permission from Ref. [34]. Copyright 2002 American Chemical Society

并且增溶能力受侧链影响较大. 随后, Kang 等 ${ }^{[35]}$ 报道 poly[ $p$-phenylene]ethynylene (PPES)与碳管是通过缠绕 而非平行的方式相互作用，并且认为 PPES 与碳管的作 用时间对 PPES 在碳管表面作用的方式影响很大(图 7). 当作用时间较短时, PPES 倾向于以平行的方式覆盖在 碳管表面; 当作用时间增加时, PPES 更倾向于以缠绕的 方式覆盖在碳管表面. 通过紫外-可见一近红外吸收光 谱, 可观察到作用时间更长时，增溶效率明显增加. Chen 等 ${ }^{[36]}$ 对比了聚苯撑乙烯和聚苯撑乙炔对不同手性 碳管的选择性(图 8), 聚合物 P $m$ PEpPE 对 $(6,5)$ 碳管有很 好的选择性，说明选择性和聚合物构型有很大关系; 而 聚合物 $\mathrm{P} m \mathrm{PV} p \mathrm{PV}$ 没有观察到选择性, 说明聚合物对碳 管的选择能力与聚合物的富电子程度相关. 对比 PmPV, PAE 与碳管有更强的 $\pi-\pi$ 相互作用，导致对碳管的增溶 能力明显提高, 并且通过改变 PAE 构型, 使其更有利于

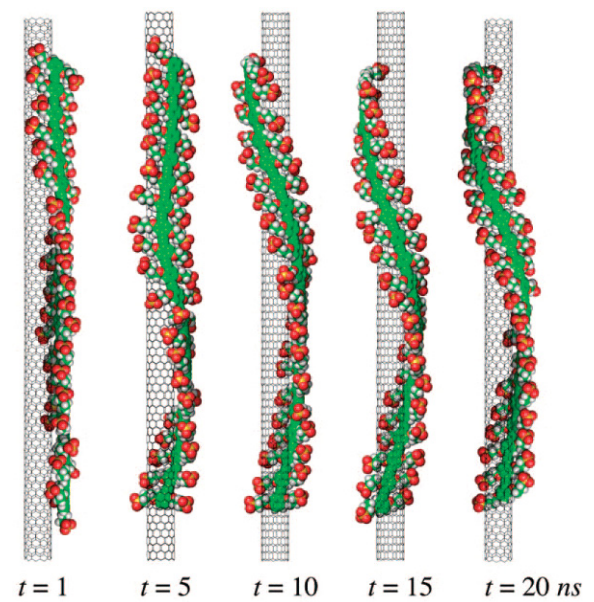

图 7 PPES 与碳管作用随时间变化分子模拟示意图 ${ }^{[35]}$

Figure 7 Snapshots of the interaction process between SWNT and PPES obtained through MD simulation ${ }^{[35]}$

Reprinted with permission from Ref. [35]. Copyright 2009 American Chemical Society 


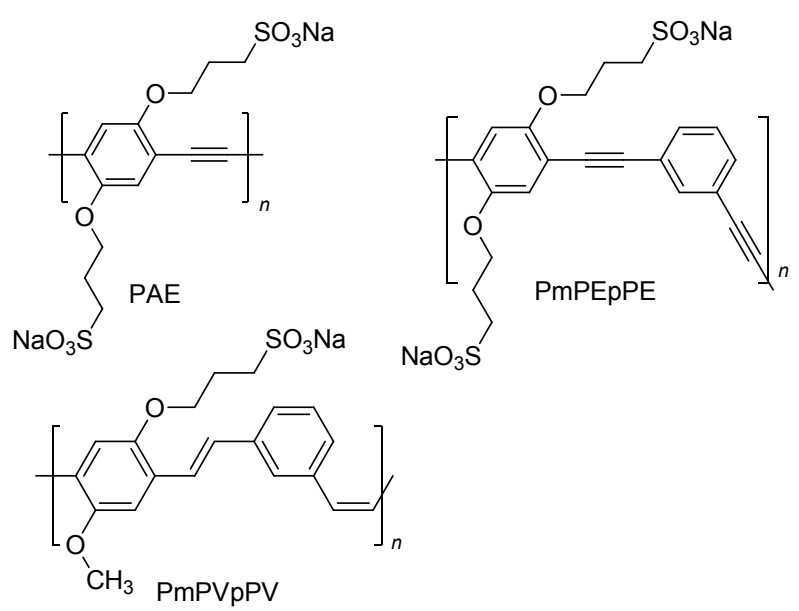

图 8 文献[36]报道的聚合物化学结构

Figure 8 Chemical structures of polymers studied in Ref. [36]

缠绕在碳管表面, 聚合物出现对特定手性碳管的选择 性. Wei 等 ${ }^{\left[{ }^{37}\right]}$ 报道了苯撑乙炔和 2-胺基嘧啶环共聚物对 半导体型碳管有很好的增溶和选择能力, 并利用三氟乙 酸对 2-胺基嘧啶单元的质子化来除去包裹碳管聚合物. 结果发现, 加入三氟乙酸能除去大部分包裹碳管的聚合 物，但是依旧有一些聚合物包覆在碳管表面而残留在体 系中. 继续添加三氟乙酸, 这些聚合物依然无法除去. 这说明聚合物与碳管的包裹是多层次的, 直接和碳管相 互作用的聚合物由于苯撑乙炔单元与碳管的相互作用 非常强很难通过 2-胺基嘧啶的质子化来解离包裹. 作者 进一步通过分子动力学模拟苯撑乙炔单元和嘧啶单元 与碳管的结合, 证实了嘧啶单元与碳管相互作用是通过 静电吸引实现的, 比较弱; 而苯撑乙炔与碳管有较强 $\pi-\pi$ 相互作用，对聚合物包裹碳管起到关键作用.

聚茄(PF) 是一类对碳管有优秀分离能力的试剂, 尤 其是对半导体性碳管有很好的选择性. 图 9 展示了含辛 基侧链的 PFO 倾向于选择直径比较小的近扶手型碳管, 特别是 $(8,6)$ 碳管 $^{[10]}$. 后续对聚芴的侧链长度进行探究, 同样发现了不同侧链长度的聚荡也倾向于选择手性角 较大的碳管(图 10$)^{[16]}$. 另外, 侧链长度足够长时对碳管 的选择变差, 这主要是侧链对碳管选择性的影响, 但能 看出, 骨架结构不变时, 在侧链影响没有达到足够大之 前, 聚合物构型对选择性来说是最关键的影响因素. 选 择性增溶是与一些种类的碳管作用较强, 与其余碳管作 用稍弱. 聚荡的选择性来源于与这些近扶手型碳管可以 实现面对面的 $\pi-\pi$ 相互作用, 从而比其他结构碳管有更 强的相互作用. 在构型上, 荡中间有一个五元环连接两 个苯环, 使得这两个苯环既不会像联苯那样在构型上随 意翻转, 又有足够的柔性扭曲成特定角度, 所以对近扶 手型的碳管选择性较好.

相比荡均聚物, 芴与其他光电基元形成的共聚物对
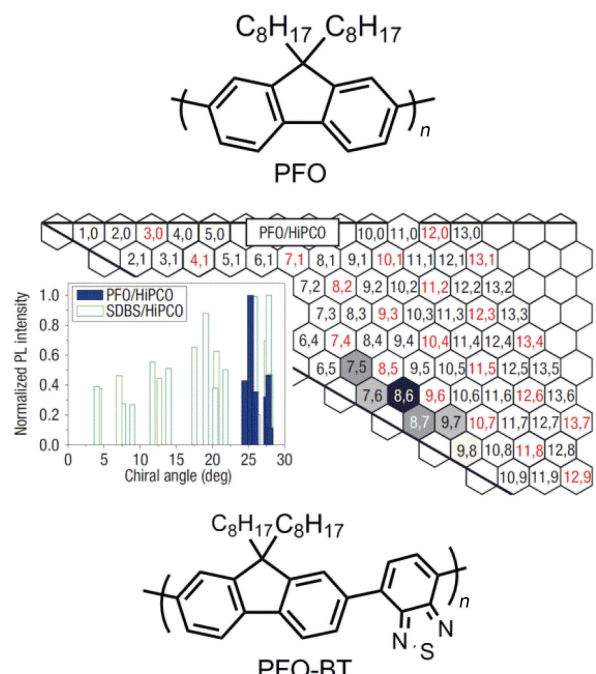

PFO-BT

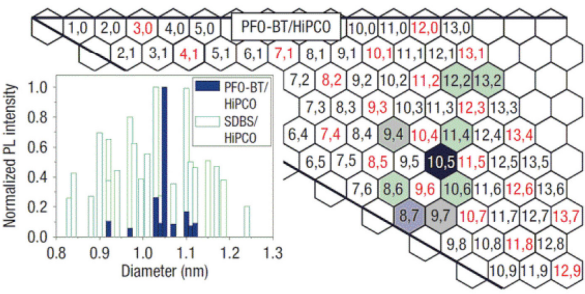

图 9 PFO 和 PFO-BT 选择性分离碳管的示意图 ${ }^{[10]}$

Figure 9 PFO and PFO-BT selectively disperse different specific chiral SWNTs ${ }^{[10]}$

Reprinted with permission from Ref. [10]. Copyright 2007 Springer Nature

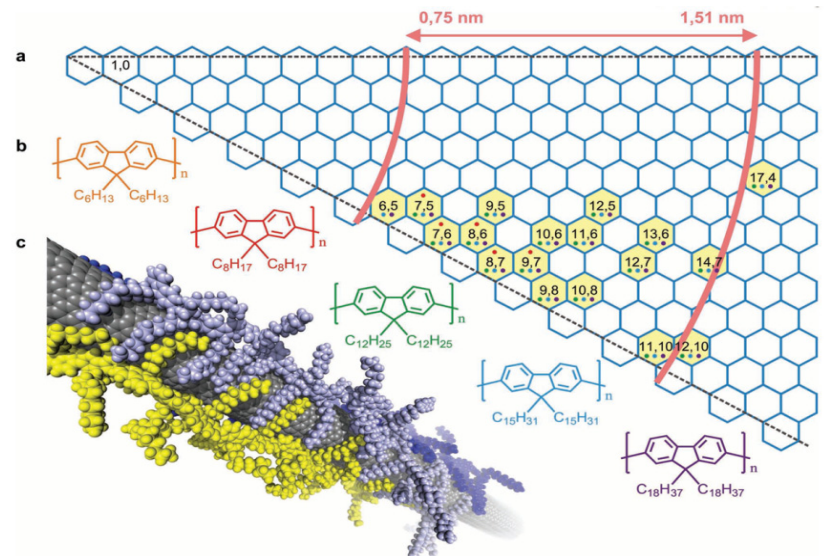

图 10 不同侧链长度的聚芴选择碳管的手性分布图 ${ }^{[16]}$

Figure 10 Chirality map of SWCNTs selected by polyfluorene wrapping ${ }^{[16]}$

In yellow the SWNTs selected are underlined; the color of the dots inside the hexagons indicates which of the polyfluorene derivatives (color code used for the chemical structures) is able to select the nanotubes. Reprinted with permission from Ref. [16]. Copyright 2013 John Wiley and Sons

碳管的选择性发生了变化. 比如, Nish 等 ${ }^{[10]}$ 报道的 PFOBT 优先选择 $(10,5)$ 碳管, 比 PFO 选择的碳管手性角要 小(图 9). PFO-BT 中的苯并噻二唑与芴是单键连接，当 聚合物缠绕在碳管表面时，苯并噻二唑单元可以找到与 


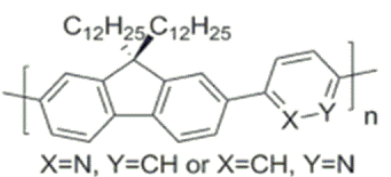

P1

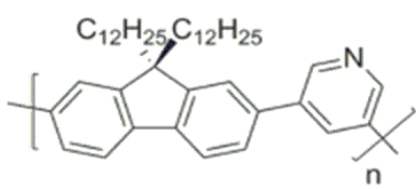

P2

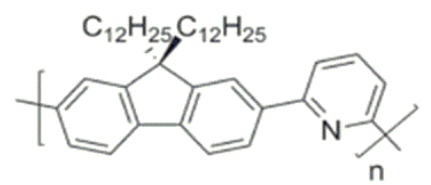

P3

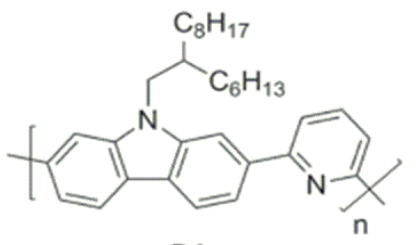

P4
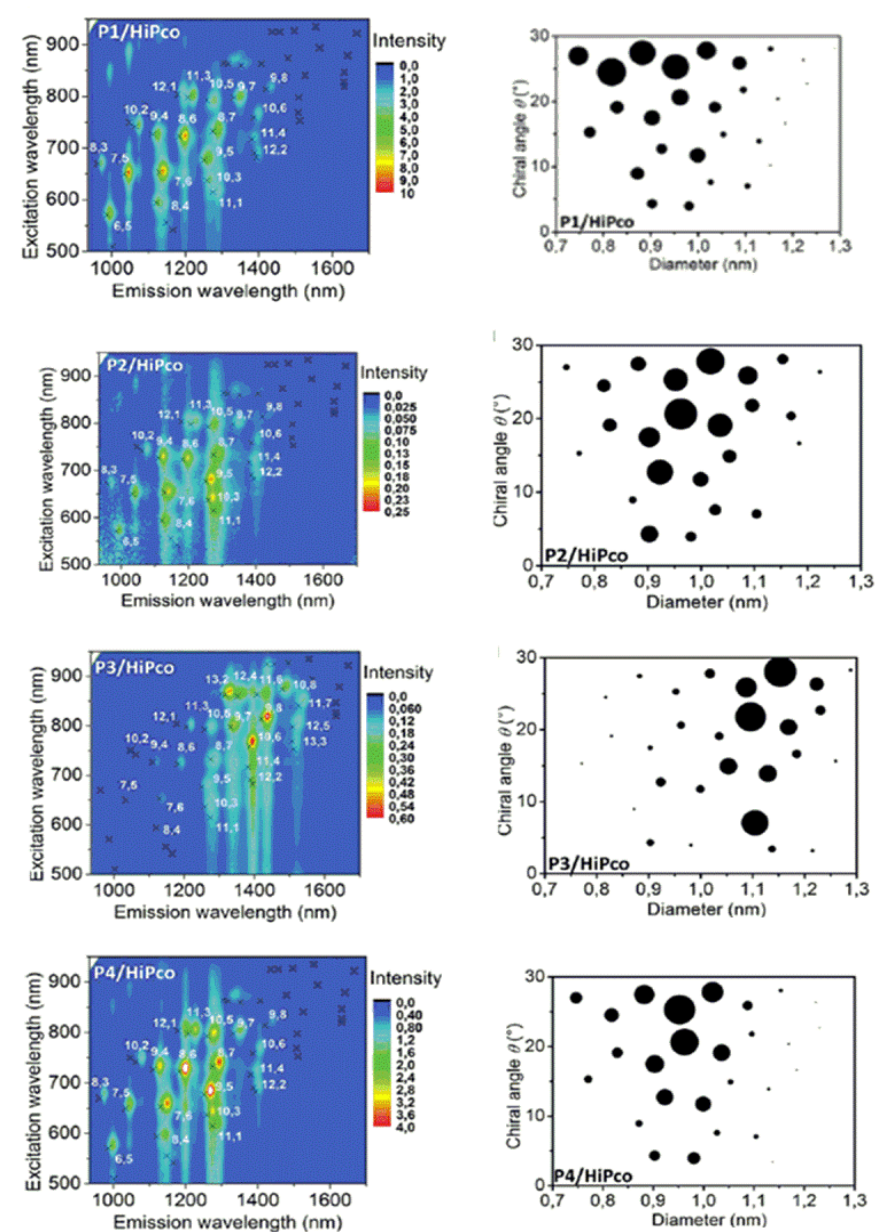

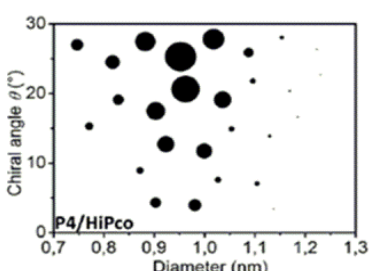

图 11 聚(芴-吡啶)和聚(咔唑-吡啶)的化学结构及其选择性分离所得碳管的手性图 ${ }^{[39]}$

Figure 11 Chemical structures of polyfluorene and polycarbazole derivatives and their separated SWNTs ${ }^{[39]}$ Reprinted with permission from Ref. [39]. Copyright 2013 John Wiley and Sons

碳管 $\pi-\pi$ 相互作用最佳的角度, 使聚合物能与碳管适配 的角度更大. 所以, 相比于 PFO, PFO-BT 对手性角较小 的碳管或者直径较大的碳管的选择性会更好. Tange 等 ${ }^{[38]}$ 报道 PFO-BT 对大直径碳管也表现出很好的选择性. Berton 等 ${ }^{[39]}$ 报道了图 11 所示的芴、咔唑与吡啶共聚物 $(P 1 、 P 2 、 P 3 、 P 4)$ 对碳管选择性分离的情况, 结果发现 这四种聚合物都可以分离多种直径较小的手性碳管, 选 择性不佳(图 11). 但对于大直径的碳管, 研究发现聚合 物中吡啶是间位连接的比对位连接的选择性更好(图 $12 \mathrm{a})$. 这是因为吡啶间位连接的聚合物可以按照特定手 性的碳管卷曲方式缠绕在碳管表面(图 12b). 另外, 在吡 啶间位连接的聚合物中, $\mathrm{N}$ 的位置对选择性也有影响。 这是由于 $\mathrm{N}$ 原子是富电子的, 当 $\mathrm{N}$ 离两边的芴更近时, 则与碳管更贴近, 导致与碳管相互作用更强, 选择性更 好. Aumaitre 等 ${ }^{[40]}$ 报道了多环芳烃蒽嵌蒽单元分别与芴 和萠单元的共聚物对碳管的增溶, 发现蒽嵌葱与葋的共 聚物对半导体型碳管有较好的富集作用, 而蒽嵌葱与萠 的共聚物对所有碳管增溶效果都较好, 导致选择性较
差，可见相比于芴结构单元，更富电子的多元芳烃与碳 管的相互作用更强，使其能对所有碳管增溶，导致选择 性不好.

聚咔唑(PC)同样被报道出具有对半导体型碳管的 选择性. 通过对比 PFO 和 PC, 发现 PC 与手性角较小的 碳管相互作用较强 ${ }^{[12]}$, 而 PFO 更倾向于选择手性角较 大的近扶手型碳管. 图 13 展示了聚咔唑和聚芴(都以十 聚体为代表)与碳管 $(10,2) 、(7,6)$ 结合后体系能量的计算 优化情况，发现聚咔唑与手性角较小的 $(10,2)$ 碳管结合 得到的体系能量较低, 而聚芴与手性角较大的 $(7,6)$ 碳 管结合得到的体系能量低. 从结构上看, 聚咔唑只有一 条长烷基侧链，而聚芴有两条长烷基侧链. 另外一个结 构区别在于五元环顶端元素, 芴基为 $\mathrm{C}$ 而咔唑为 $\mathrm{N}$ 元 素，因此这两方面都可能是聚合物表现出对不同手性的 碳管选择性不同的因素. 但 Fukumaru 等 ${ }^{[41]}$ 发现，聚芴 上不同数量烷基侧链对 HiPCO 碳管选择性的影响不大 (图 14), 由此可见聚咔唑中 $\mathrm{N}$ 的引入对聚合物与碳管结 合的构象和结合后体系能量影响较大. 
(a)

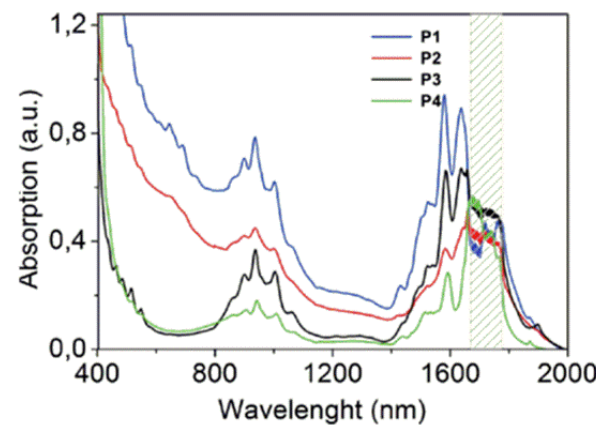

(b)

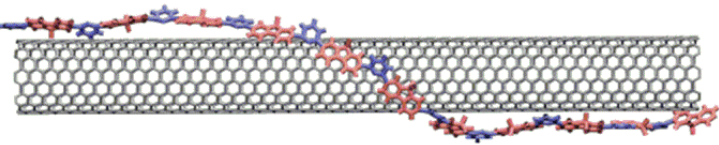

图 12 (a) P1, P2, P3, P4 对大直径碳管选择性分离后所得碳 管溶液的紫外可见近红外吸收光谱图和(b)聚合物 P3 在碳管 表面缠绕的示意图 ${ }^{[39]}$

Figure 12 (a) UV-vis-NIR light absorption spectra of the solutions of separated SWNTs by polymer P1, P2, P3, P4, and (b) schematic diagram of $\mathbf{P 3}$ wrapping on the surface of SWNT ${ }^{[39]}$ In (b) Red part represents fluorene unit, while blue rings are pyridine units ${ }^{[39]}$. Reprinted with permission from Ref. [39]. Copyright 2013 John Wiley and Sons

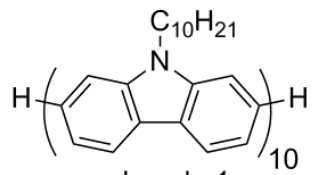

carbazole 1

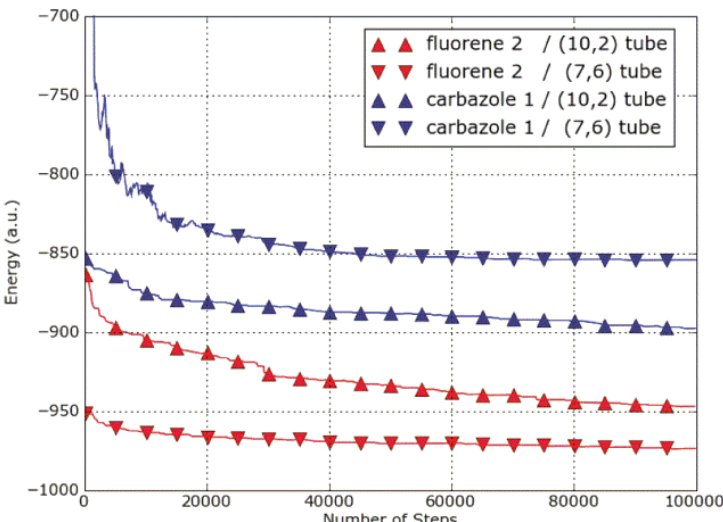

图 13 荡、咔唑十聚体与 $(10,2)$ 、 $(7,6)$ 碳管结合后体系能量 的计算优化过程 ${ }^{[12]}$

Figure 13 Computing optimization on energies of polymer/SWNT complexes formed from binding $(10,2)$ and $(7,6)$ SWNTs with fluorene decamer and carbazole decamer ${ }^{[12]}$

Reprinted with permission from Ref. [12]. Copyright 2011 American Chemical Society

\section{3 杂环主链的刚性聚合物}

除了以苯环为主的刚性聚合物, 聚噻吩这种五元杂 环的聚合物与碳管的相互作用也很强. Foroutan 等 ${ }^{[42]}$ 通过分子动力学模拟，发现聚合物与碳管的相互作用强 弱顺序为 $\mathrm{PT}>\mathrm{PPyPV}>\mathrm{PPy}>\mathrm{PmPV}$ (图 15), 可以看出 五元杂环与碳管的相互作用比苯环更强. Stranks 等 ${ }^{[43]}$ 报 道了聚芴包裹的碳管能完全被聚噻吩替换, 形成聚噻吩 包裹的碳管，证明了聚噻吩与碳管的相互作用比聚荡 强. 相比于苯环, 噻吩与碳管的作用角度更小, 即与碳 管更贴合，导致其作用强度更大. Imin 等 ${ }^{[44]}$ 报道了聚 芴、聚噻吩和芴与噻吩的共聚物对碳管的增溶，发现聚 合物的紫外-可见吸收峰在相应的复合材料中发生了红 移，这是由于吸附在碳管表面后，聚合物主链的平面化 所致, 而聚噻吩的红移位移明显比其他两个聚合物的 大，证明聚噻吩与碳管的相互作用更强. Lee 等 ${ }^{[11]}$ 报道 了噻吩只有在烷基侧链长度到达 12 个碳时才表现出选 择性，当烷基侧链较短或密度不够时，增溶能力都不强.

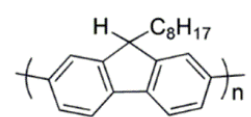

C8H-PF

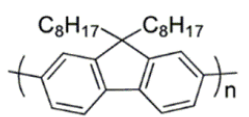

2C8-PF

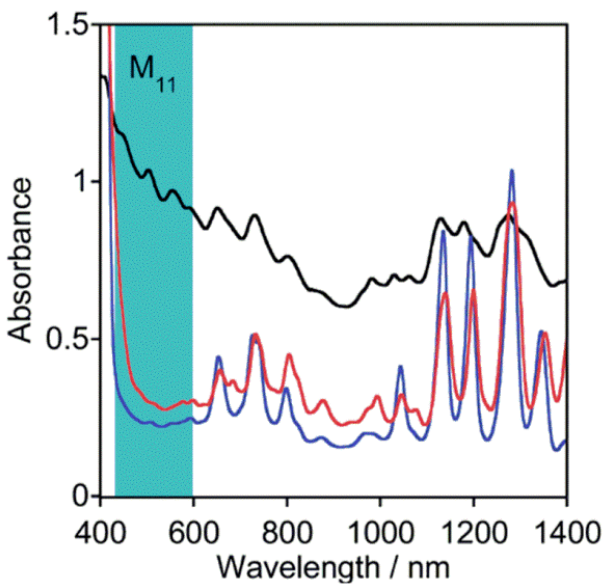

图 $14 \mathrm{C} 8 \mathrm{H}-\mathrm{PF}$ (红)和 $2 \mathrm{C} 8 \mathrm{-PF}$ (兰)的化学结构和及其分离所得 碳管的吸收光谱 ${ }^{[39]}$

Figure 14 Chemical structures of C8H-PF (red) and 2C8-PF (blue) and light-absorption spectra of their separated SWNTs ${ }^{[39]}$ Reprinted with permission from Ref. [39]. Copyright 2014 Royal Society of Chemistry

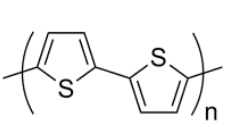

PT<smiles></smiles>
PPy<smiles>CCCOc1cc(C=Cc2cccc(C=CC(C)C)c2)c(OCC)cc1C</smiles>

PmPV

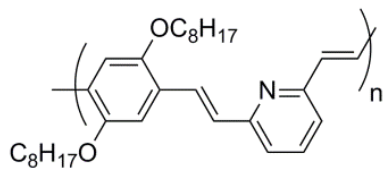

PPyPV

图 15 文献[42]研究的聚合物化学结构

Figure 15 Chemical structures of the polymers studied in Ref. [42] 
这是因为聚噻吩的刚性更强, 与碳管相互作用时以近乎 平行的方式覆盖在碳管表面, 这时侧链的长度成为影响 聚合物与碳管相互作用的关键因素. 这与聚苯撑乙炔比 较相似. Wang 等 ${ }^{[45}$ 报道了聚噻吩随着烷基侧链长度增 加, 增溶强度增加; Lee 等 ${ }^{[46]}$ 发现聚噻吩烷基链密度降 低, 增溶能力明显下降. Wang 等 ${ }^{[45}$ 提出聚噻吩对碳管 的选择性机理是聚合物包裹的金属型碳管极化率高, 导 致在非极性溶剂中聚集, 生成沉淀, 实现对半导体型碳 管的选择性分离. Gomulya 等 ${ }^{[47}$ 报道了两种主链引入亚 胺基元的噻吩共聚物(图 16), 发现 PAMDD 对半导体型 碳管的选择性比 PAZDD 好, 该作者认为亚胺基元的引 入使聚合物对碳管的吸附能力增加, 而 PAZDD 柔性比 PAMDD 要好, 导致聚合物对金属型碳管的增溶能力增 加, 选择性变差; 同时 PAMDD 中的苯环缓解了由于亚 胺基元导致的对碳管吸附的影响. Lei 等 ${ }^{[88]}$ 对比了六种 以 diketopyrrolopyrrole (DPP)为骨架的聚合物, 发现增 加噻吩单元, 提高聚合物对半导体型碳管的增溶效率. Min 等 ${ }^{[49]}$ 同样观察到在以 binaphthyl 为骨架的聚合物中, 增加噻吩单元个数能提高聚合物对半导体型碳管的增 溶效率.

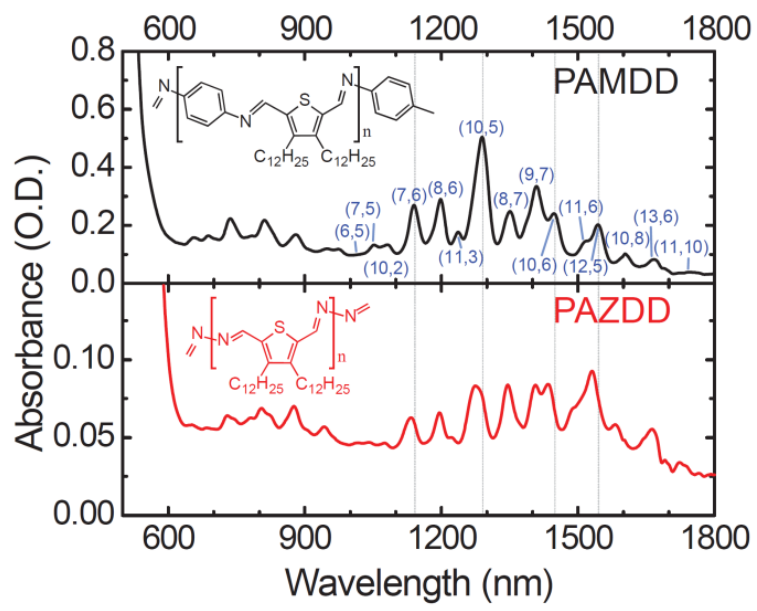

图 16 含亚胺的噻吩聚合物及其分离得到碳管溶液的吸收光 谱 ${ }^{[47]}$

Figure 16 Imine-containing thiophene polymers and light-absorption spectra of their separated SWNT solutions ${ }^{[47]}$

Reprinted with permission from Ref. [47]. Copyright 2015 John Wiley and Sons

同时, 对导电性碳管有选择性的还有 $\mathrm{DNA}^{[50]}$ 、 $\mathrm{RNA}^{[51]}$ 和卟啉 ${ }^{[52]}$ 等几种常见的结构, 都是含有五元杂 环结构. 2003 年, Zheng 等 ${ }^{[50]}$ 报道了 ssDNA 可以通过 $\pi-\pi$ 相互作用缠绕在碳管表面, 实现对碳管的增溶. 随后, 他们还报道了 ssDNA 能选择性分离金属型和半导体型 碳管，认为选择机理源自金属型和半导体型碳管的不同 极化率 ${ }^{[9]}$. Zaremba 等 ${ }^{[11]}$ 报道了 tRNA 对直径较小的 CoMoCAT 碳管和 HiPCO 都有较好的增溶能力. Yan
等 ${ }^{[53]}$ 报道了肝素钠对直径较大的碳管有较好的选择性 (图 17), 并且与碳管 $(8,4)$ 结合能最高. Murakami 等 ${ }^{[5]}$ 报 道了卟啉对碳管的增溶能力，并发现卟啉中间的金属离 子对增溶影响不大. 随后 $\mathrm{Li}$ 等 ${ }^{[52]}$ 发现卟啉对半导体型 碳管有较好的选择性.

(a)

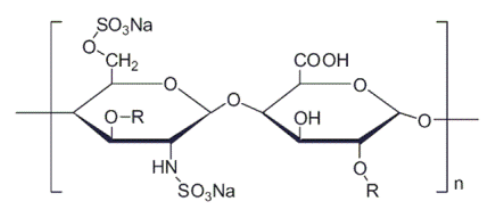

(b)

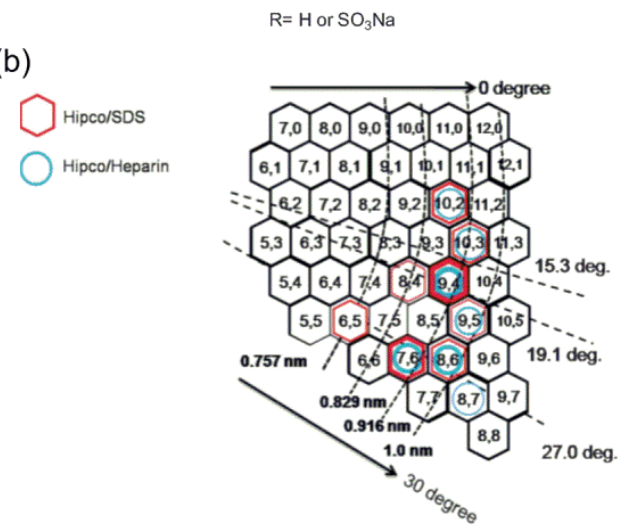

图 17 (a)肝素钠的化学结构式和(b)肝素钠对不同手性碳管的 选择 ${ }^{[53]}$

Figure 17 (a) Chemical structure of heparin sodium, and (b) different chiral SWNTs sorted by heparin sodium ${ }^{[53]}$

Reprinted with permission from Ref. [53]. Copyright 2010 John Wiley and Sons

\section{3 侧链长度}

聚合物侧链对碳管选择性增溶的影响一直是研究 的热点, 尤其是聚苆和聚噻吩的衍生物, 不同长度烷基 侧链表现出对碳管不同程度的选择性. 聚荡在烷基侧链 长度增加时, 选择性变差. 这是由于聚荡中的烷基侧链 同前文所述的烷基链一样，与碳管之间存在着相互作 用，会覆盖在碳管表面. 但这种相互作用和覆盖，没有 手性选择性. 当烷基侧链长度增加之后, 侧链对碳管的 覆盖率提高, 会导致对所有碳管无差别的增溶, 导致选 择性变差. 可见，聚合物烷基侧链的长度对碳管分离的 选择性影响主要来源于其在碳管上的覆盖率大小。但 是, 也有覆盖率理论意外的例子. 比如, Fukumaru 等 ${ }^{[41]}$ 报道了比 PFO 在芴上少一条烷基侧链的聚芴结构 C8H-PF，根据上面提到的覆盖率理论，C8H-PF 的烷基 侧链密度较低, 一定会影响聚芴对碳管的增溶效果. 但 研究发现, C8H-PF 与 PFO 相比, 对 HiPCO 这种小直径 的碳管选择性和增溶能力差别不大. 这可能是因为聚芴 对直径较小碳管的亲和力较强, 侧链覆盖率对此影响力 有限, 但对于大直径碳管, 这两种聚合物的增溶能力差 
别较大, 图 18 所示 PFO-SWNT 的 1/6 的吸光强度比 C8H-PF-SWNT 的吸光强度要强, 这无疑是烷基侧链的 覆盖率起到了作用. 说明选择性增溶是一种强度刚好的 增溶, 增溶能力过强一定会导致选择性下降. C8H-PF 由 于烷基侧链密度降低, 对碳管覆盖率降低, 实现了一种 对大直径碳管刚好的增容效果, 即选择性增溶. Gifford 等 ${ }^{[55]}$ 通过力场计算发现, 当烷基侧链长度为 8 个碳或更 长时，聚荡主链缠绕在碳管表面的几何构型会因侧链发 生改变, 导致聚合物主链与碳管的 $\pi-\pi$ 相互作用减弱, 而烷基侧链和碳管的范德华力相互作用增强, 实现了对 不同导电性的碳管的区分和选择性增溶. Lee 等 ${ }^{[11]}$ 观察 到聚噻吩烷基侧链变长导致对碳管的选择性变好(图 19). 因为聚噻吩刚性太强, 使其对碳管的细绕不如聚

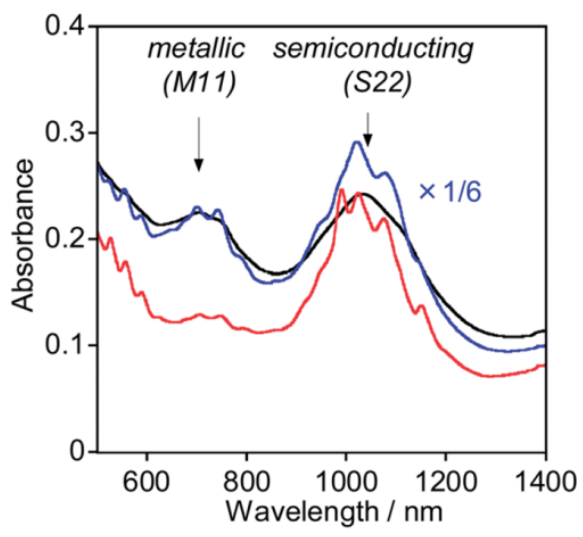

图 $18 \mathrm{C} 8 \mathrm{H}-\mathrm{PF}$ (红色)和 2C8-PF(蓝色)对大直径碳管选择性分 离的吸收光谱 ${ }^{[41]}$

Figure 18 Light-absorption spectra of SWNT solution sorted by C8H-PF (red) and 2C8-PF (blue) ${ }^{[41]}$

Reprinted with permission from Ref. [41]. Copyright 2014 Royal Society of Chemistry

(a)

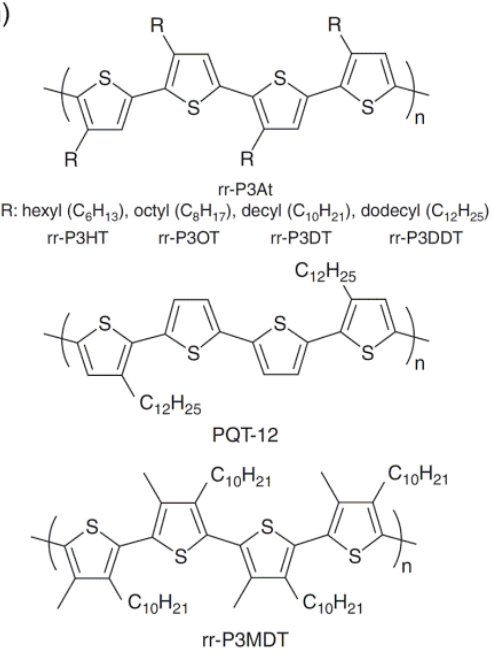

芴紧密, 所以聚噻吩对碳管的增溶需要较高的烷基侧链 覆盖率才能实现.

总之, 烷基侧链对聚合物选择性增溶碳管的影响是 覆盖率起作用. 因此, 烷基侧链的覆盖率可作为聚合物 对碳管选择性增溶的影响因素之一. 对于聚荡这种与碳 管适配性高的聚合物，烷基侧链不能过长，不然会导致 对碳管无差别增溶，降低选择性，而对于聚噻吩这种与 碳管适配性不高的聚合物, 烷基侧链增长, 会一起帮助 噻吩骨架实现对特定手性碳管的选择.

\section{4 温度}

温度会对聚合物和碳管相互作用的动力学产生影 响. 当温度较高时, 整个体系比较活跃, 增溶效果增强. 对于聚芴这种与碳管相互作用较强的聚合物结构, 温度 太高会导致聚合物活跃度过高，对碳管无差别增溶，降 低选择性. 李清文等 ${ }^{[56]}$ 报道了聚荡随着体系温度升高, 对碳管选择性降低(图 20a), 实验证实了上述推断. 对于 聚噻吩这种与碳管相互作用强的聚合物结构, 温度一定 要稍高，不然聚合物活跃度不够，增溶效果不好。如 Lee 等 ${ }^{[11]}$ 研究聚噻吩选择性的同时, 探究了从 $-40{ }^{\circ} \mathrm{C}$ 到 $90{ }^{\circ} \mathrm{C}$ 聚噻吩的增溶效果, 发现在 $50{ }^{\circ} \mathrm{C}$ 效果最佳. 相 比于聚芴体系表现出选择性的合适温度为 $10{ }^{\circ} \mathrm{C}$ 以内, $50{ }^{\circ} \mathrm{C}$ 算是较高的温度. Gomulya 等 ${ }^{[57]}$ 报道 poly(3-dodecylthiophene-2,5-diyl) (P3DDT) 在 $50{ }^{\circ} \mathrm{C}$ 下溶于溶剂, 排除了聚合物在低温下溶解度低，导致不能与碳管充分 作用的影响，发现 $10 \sim 20{ }^{\circ} \mathrm{C}$ 时聚合物对碳管的分散能 力最强(图 20b), 这与之前报道的聚芴选择性增溶碳管 的条件相似，在较长的超声条件下，温度升高，溶液中

(b)

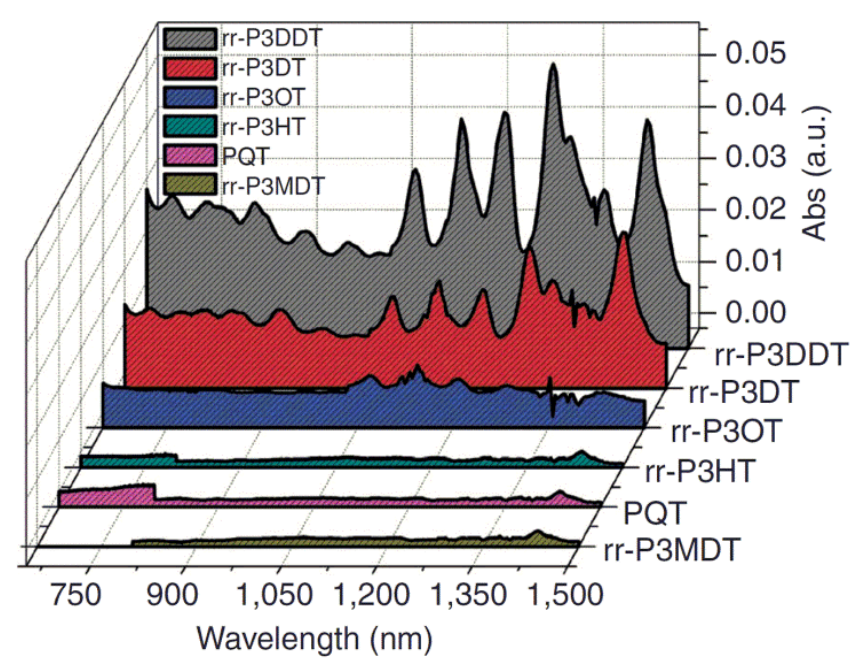

图 19 (a)具有不同烷基侧链聚噻吩的化学结构式和(b)由此分离所得碳管溶液的吸收光谱 ${ }^{[11]}$

Figure 19 (a) Chemical structures of polythiophenes bearing different alkyl side chains, and (b) light-absorption spectra of SWNT solutions sorted therewith ${ }^{[11]}$

Reprinted with permission from Ref. [11]. Copyright 2011 Springer Nature 

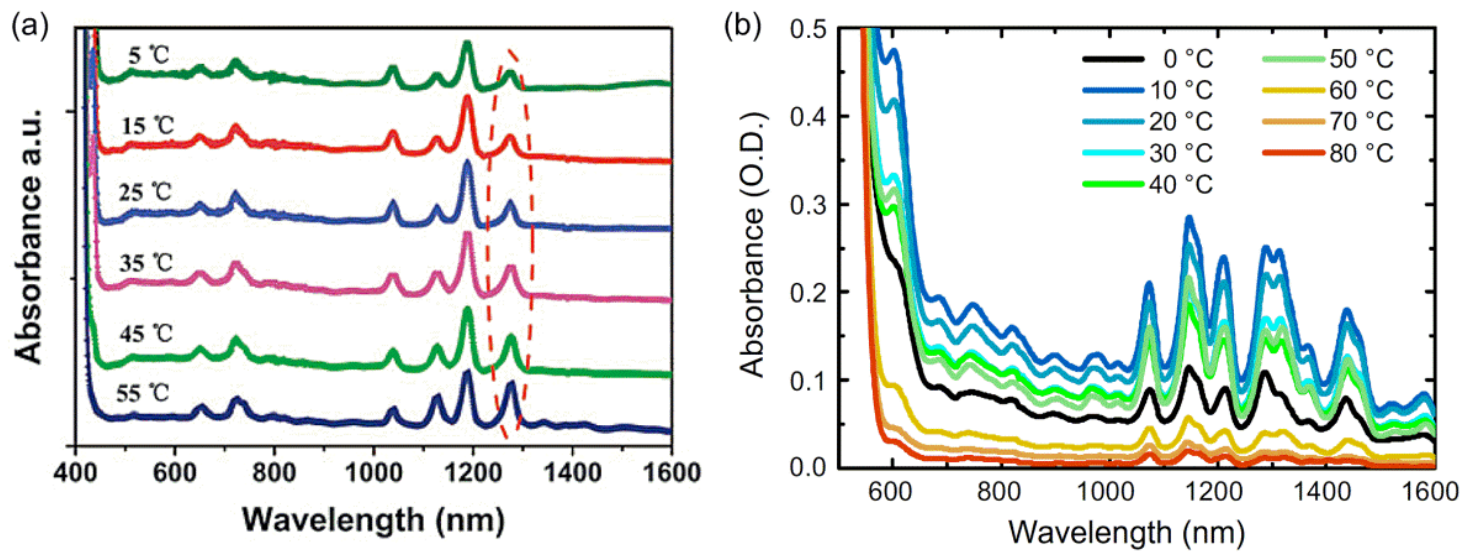

图 20 (a)不同温度下 PFO 分离的碳管溶液的吸收光谱 ${ }^{[66]}$ 和(b)不同温度下 P3DDT 分离的碳管溶液吸收光谱 ${ }^{[57]}$

Figure 20 (a) Light-absorption spectra of SWNT solutions obtained by PFO sorting at various temperatures, ${ }^{[56]}$ and (b) light-absorption spectra of SWNT solutions obtained by P3DDT sorting at various temperatures ${ }^{[57]}$

(a) Reprinted with permission from Ref. [56]. Copyright 2015 Royal Society of Chemistry. (b) Reprinted with permission from Ref. [57]. Copyright 2015 Elsevier

分散的碳管浓度下降 ${ }^{[58]}$. 可见，在聚合物与碳管相互作 用的过程, 温度通过影响聚合物的构型、聚集程度、活 动能力、和碳管相互作用强弱等, 实现聚合物对碳管增 溶能力与选择性增溶能力的影响. 而聚荡和聚噻吩在 $10{ }^{\circ} \mathrm{C}$ 的超声温度下, 增溶能力最强, 可能是因为在实 现热力学平衡的状态下, $10{ }^{\circ} \mathrm{C}$ 是聚合物与碳管 $\pi-\pi$ 相互 作用最强的条件. 总之, 温度对聚合物选择性增溶碳管 的影响, 依然是对聚合物构型这一主要影响因素的补 充. 正因为这样, 才导致不同骨架结构的聚合物在选择 性增溶碳管时最适温度有差异.

\section{5 聚合物分子量和聚合物与碳管比例}

聚合物分子量和聚合物与碳管的比例对选择性增 溶的影响原因都是聚合物在碳管表面的覆盖率. 这种外 部环境和聚合物烷基侧链的影响是不同的, 烷基侧链对 选择性增溶的影响更大, 因为烷基侧链直接参与碳管相 互作用. 聚合物分子量过低, 增溶能力不够; 聚合物分 子量过高, 聚合物溶解度可能降低, 或者对碳管增溶能 力过强, 出现无差别增溶, 选择性变差. Jakubka 等 ${ }^{[59]}$ 报 道了 PFO-BT 在不同聚合物分子量下的选择性, 发现分 子量过高或过低时, 聚合物对碳管的选择性都不好. 而 聚合物与碳管的比例是显而易见对选择性有影响的环 境因素, 比例太高和太低都容易导致选择性不好 ${ }^{[60]}$. 很 多报道聚合物对碳管选择性分离的文献中都有涉及探 究不同的聚合物与碳管比例 ${ }^{[61-62]}$.

\section{6 溶剂}

溶剂对整个分散体系的稳定性有至关重要的影响. 首先, 溶剂对聚合物要能溶解但又不能完全溶解. 这是
因为如果溶剂对聚合物溶解太好，聚合物在溶液中会有 很多构象, 不利于与碳管形成稳定的构型. Hwang 等 ${ }^{[63]}$ 通过比较几种聚荡衍生物在几种溶剂下的选择性, 得出 溶剂极性不可太高的原因是不能让聚合物在溶剂中太 自由, 导致选择性变差. 其次, Fang 等 ${ }^{[18]}$ 提出聚合物对 导电性碳管的选择与极化率有关，聚合物包裹的金属型 碳管极化率高，导致在非极性溶剂中容易聚集，生成沉 淀，而极性溶剂更容易稳定这种极化率高的碳管，导致 选择性不好.

\section{7 结论}

聚合物增溶和选择性增溶碳管是复杂的过程，影响 因素很多. 本综述从聚合物骨架、侧链长度、分子量以 及温度、聚合物/碳管比例、溶剂等方面，根据现有的研 究范例讨论其基本的影响规律，获得了以下几点认识: (1)聚合物对碳管的增溶和选择性增溶是通过 $\pi-\pi$ 相互作 用实现，聚合物主链结构的富电子程度是影响聚合物与 碳管相互作用强度的首要因素. (2)能对碳管选择性分离 的聚合物多是通过螺旋缠绕的构象与碳管相互作用, 可 以推断螺旋缠绕的构象使得聚合物与碳管整体体系的 能量最低. 因此, 聚合物主链结构能否以螺旋缠绕的构 象覆盖在碳管表面，是另一个影响聚合物对碳管选择性 增溶的关键因素. (3)聚合物与碳管相互作用时, 聚合物 烷基侧链并非作为提高聚合物在溶剂中的溶解度或亲 疏水性伸展在溶剂中存在，而多是通过范德华力与碳管 相互作用覆盖在碳管表面. 虽然范德华力的强度低于 $\pi-\pi$ 相互作用，但聚合物侧链可以通过表面覆盖率的大 小影响到聚合物对不同导电性碳管的选择性增溶能力. 因此, 在设计碳管分离聚合物时, 聚合物的烷基侧链是 对共轭主链结构非常重要的补充, 不可被忽视的. (4)其 
他外部条件, 包括温度、溶剂、聚合物/碳管比等, 都是 通过改变聚合物与碳管的体系环境来影响聚合物与碳 管相互作用强度, 实现对聚合物碳管增溶或选择性增溶 能力的影响. 虽然这些认识还存在片面性, 并没有完全 总结全部影响因素和行为规律, 但应该反映了聚合物对 碳管增溶和选择性增溶的最主要方面, 在今后聚合物分 离体系的设计和研究上加以考虑将大有裨益.

\section{References}

[1] Iijima, S. Nature 1991, 354, 56.

[2] Carlson, L. J.; Krauss, T. D. Acc. Chem. Res. 2008, 41, 235.

[3] Zhang, H.; Wu, B.; Hu, W.; Liu, Y. Chem. Soc. Rev. 2011, 40, 1324.

[4] Wang, H.; Wang, Y.; Tee, B. C. K.; Kim, K.; Lopez, J.; Cai, W.; Bao, Z. Adv. Sci. 2015, 2, 1500103.

[5] Ha, M.; Xia, Y.; Green, A. A.; Zhang, W.; Frisbie, C. D. ACS Nano 2010, 8,4388

[6] Chae, S. H.; Yu, W. J.; Bae, J. J.; Dinh Loc, D.; Perello, D.; Jeong, H. Y.; Quang Huy, T.; Thuc Hue, L.; Quoc An, V.; Yun, M.; Duan, X.; Lee, Y. H. Nat. Mater. 2013, 12, 403.

[7] Arnold, M. S.; Green, A. A.; Hulvat, J. F.; Stupp, S. I.; Hersam, M. C. Nat. Nanotechnol. 2006, 1, 60.

[8] Krupke, R.; Hennrich, F.; Lohneysen, H.; Kappes, M. M. Science 2003, 301, 344.

[9] Zheng, M.; Jagota, A.; Strano, M. S.; Santos, A. P.; Barone, P.; Chou, S. G.; Diner, B. A.; Dresselhaus, M. S.; Mclean, R. S.; Onoa, G. B.; Samsonidze, G. G.; Semke, E. D.; Usrey, M.; Walls, D. J. Science 2003, 1545 .

[10] Nish, A.; Hwang, J. Y.; Doig, J.; Nicholas, R. J. Nat. Nanotechnol. 2007, 2, 640 .

[11] Lee, H. W.; Yoon, Y.; Park, S.; Oh, J. H.; Hong, S.; Liyanage, L. S.; Wang, H.; Morishita, S.; Patil, N.; Park, Y. J.; Park, J. J.; Spakowitz, A.; Galli, G.; Gygi, F.; Wong, P. H.; Tok, J. B.; Kim, J. M.; Bao, Z. Nat. Commun. 2011, 2, 541.

[12] Lemasson, F. A.; Strunk, T.; Gerstel, P.; Hennrich, F.; Lebedkin, S.; Barner-Kowollik, C.; Wenzel, W.; Kappes, M. M.; Mayor, M. J. Am. Chem. Soc. 2011, 133, 652.

[13] O'Connell, M. J.; Bachilo, S. M.; Huffman, C. B.; Moore, V. C.; Strano, M. S.; Haroz, E. H.; Rialon, K. L.; Boul, P. J.; Noon, W. H.; Kittrell, C.; Ma, J.; Hauge, R. H.; Weisman, R. B.; Smalley, R. E. Science 2002, 297, 593.

[14] Itkis, M. E.; Perea, D. E.; Jung, R.; Niyogi, S.; Haddon, R. C. J. Am. Chem. Soc. 2005, 127, 3439.

[15] Shaffer, M. S. P.; Windle, A. H. Adv. Mater. 1999, 11, 937.

[16] Gomulya, W.; Costanzo, G. D.; de Carvalho, E. J.; Bisri, S. Z.; Derenskyi, V.; Fritsch, M.; Frohlich, N.; Allard, S.; Gordiichuk, P.; Herrmann, A.; Marrink, S. J.; dos Santos, M. C.; Scherf, U.; Loi, M. A. Adv. Mater. 2013, 25, 2948.

[17] Wang, H.; Bao, Z. Nano Today 2015, 10, 737.

[18] Fong, D.; Adronov, A. Chem. Sci. 2017, 8, 7292.

[19] Vaisman, L.; Wagner, H. D.; Marom, G. Adv. Colloid Interface Sci. 2007, 128-130, 37.

[20] Di Crescenzo, A.; Di Profio, P.; Siani, G.; Zappacosta, R.; Fontana, A. Langmuir 2016, 32, 6559.

[21] Shvartzman-Cohen, R.; Levi-Kalisman, Y.; Nativ-Roth, E.; Yerushalmi-Rozen, R. Langmuir 2004, 20, 6085.

[22] Yudasaka, M.; Zhang, M.; Jabs, C.; Iijima, S. Appl. Phys. A: Mater. Sci. Process. 2000, 71, 449.

[23] Di Crescenzo, A.; Aschi, M.; Fontana, A. Macromolecules 2012 , 45,8043 .
[24] Yurekli, K.; Mitchell, C. A.; Krishnamoorti, R. J. Am. Chem. Soc. 2004, 126, 9902

[25] Didenko, V. V.; Moore, V. C.; Baskin, D. S.; Smalley, R. E. Nano Lett. 2005, 5, 1563.

[26] Manivannan, S.; Jeong, I. O.; Ryu, J. H.; Lee, C. S.; Kim, K. S.; Jang, J.; Park, K. C. J. Mater. Sci.: Mater. Electron. 2009, 20, 223.

[27] Pasquinelli, S. S. T. a. M. A. J. Phys. Chem. B 2010, 114, 4122.

[28] Curran, S. A.; Ajayan, P. M.; Blau, W. J.; Carroll, D. L.; Coleman, J. N.; Dalton, A. B.; Davey, A. P.; Drury, A.; McCarthy, B.; Maier, S.; Strevens, A. Adv. Mater. 1998, 10, 1091.

[29] Coleman, J. N.; Dalton, A. B.; Curran, S.; Rubio, A.; Davey, A. P.; Drury, A.; McCarthy, B.; Lahr, B.; Ajayan, P. M.; Roth, S.; Barklie, R. C.; Blau, W. J. Adv. Mater. 2000, 12, 213.

[30] Dalton, A. B.; Stephan, C.; Coleman, J. N.; McCarthy, B.; Ajayan, P. M.; Lefrant, S.; Bernier, P.; Blau, W. J.; Byrne, H. J. J. Phys. Chem. B 2000, 104, 10012.

[31] Yi, W.; Malkovskiy, A.; Xu, Y.; Wang, X.-Q.; Sokolov, A. P.; Lebron-Colon, M.; Meador, M. A.; Pang, Y. Polymer 2010, 51, 475.

[32] In het Panhuis, M.; Maiti, A.; Dalton, A. B.; van den Noort, A.; Coleman, J. N.; McCarthy, B.; Blau, W. J. J. Phys. Chem. B 2003, 107, 478.

[33] Yi, W.; Malkovskiy, A.; Chu, Q.; Sokolov, A. P.; Colon, M. L.; Meador, M.; Pang, Y. J. Phys. Chem. B 2008, 112, 12263.

[34] Chen, J.; Liu, H.; Weimer, W. A.; Halls, M. D.; Waldeck, D. H.; Walker, G. C. J. Am. Chem. Soc. 2002, 124, 9034.

[35] Kang, Y. K.; Lee, O.-S.; Deria, P.; Kim, S. H.; Park, T.-H.; Bonnell, D. A.; Saven, J. G.; Therien, M. J. Nano Lett. 2009, 9, 1414.

[36] Chen, Y.; Xu, Y.; Wang, Q.; Gunasinghe, R. N.; Wang, X. Q.; Pang, Y. Small 2013, 9, 870.

[37] Wei, X.; Maimaitiyiming, X. Macromol. Chem. Phys. 2020, 221.

[38] Tange, M.; Okazaki, T.; Iijima, S. J. Am. Chem. Soc. 2011, 133, 11908.

[39] Berton, N.; Lemasson, F.; Poschlad, A.; Meded, V.; Tristram, F.; Wenzel, W.; Hennrich, F.; Kappes, M. M.; Mayor, M. Small 2014, 10,360 .

[40] Aumaitre, C.; Fong, D.; Adronov, A.; Morin, J.-F. Polym. Chem. 2019, 10, 6440.

[41] Fukumaru, T.; Toshimitsu, F.; Fujigaya, T.; Nakashima, N. Nanoscale 2014, 6, 5879.

[42] Foroutan, M.; Nasrabadi, A. T. J. Phys. Chem. B 2010, 114, 5320.

[43] Stranks, S. D.; Habisreutinger, S. N.; Dirks, B.; Nicholas, R. J. Adv. Mater. 2013, 25, 4365.

[44] Imin, P.; Cheng, F.; Adronov, A. Polym. Chem. 2011, 2, 411.

[45] Wang, H.; Koleilat, G. I.; Liu, P.; Jiménez-Osés, G.; Lai, Y.-C.; Vosgueritchian, M.; Fang, Y.; Park, S.; Houk, K. N.; Bao, Z. ACS Nano 2014, 8, 2609 .

[46] Lee, M.-H.; Lee, S.-H.; Kim, J.; Lee, S. Y.; Lim, D.-H.; Hwang, K.; Hwang, H.; Jung, Y. C.; Noh, Y.-Y.; Kim, D.-Y. Carbon 2017, 125, 571.

[47] Gomulya, W.; Derenskyi, V.; Kozma, E.; Pasini, M.; Loi, M. A. Adv. Funct. Mater. 2015, 25, 5858

[48] Lei, T.; Pitner, G.; Chen, X.; Hong, G.; Park, S.; Hayoz, P.; Weitz, R. T.; Wong, H.-S. P.; Bao, Z. Adv. Electron. Mater. 2016, 2, 1500299.

[49] Min, S. H.; Kim, H.-I.; Kim, K.-S.; Cha, I.; Ha, S.; Yun, W. S.; Kwak, S. K.; Kim, J.-H.; Kim, B.-S.; Song, C. Polymer 2016, 96, 63.

[50] Zheng, M.; Jagota, A.; Semke, E. D.; Diner, B. A.; McLean, R. S.; Lustig, S. R.; Richardson, R. E.; Tassi, N. G. Nat. Mater. 2003, 2, 338.

[51] Zaremba, O.; Goldt, A.; Ramirez-Morales, M.; Khabushev, E. M.; Shulga, E.; Eremin, T.; Prikazchikova, T.; Orekhov, A.; Grebenko, A.; Zatsepin, T. S.; Obraztsova, E. D.; Nasibulin, A. G. Carbon 
2019, 151, 175 .

[52] Li, H.; Zhou, B.; Lin, Y.; Gu, L.; Wang, W.; Fernando, K. A. S.; Kumar, S.; Allard, L. F.; Sun, Y.-P. J. Am. Chem. Soc. 2004, 126, 1014.

[53] Yan, L. Y.; Li, W.; Fan, X. F.; Wei, L.; Chen, Y.; Kuo, J.-L.; Li, L.-J.; Kwak, S. K.; Mu, Y.; Chan-Park, M. B. Small 2010, 6, 110.

[54] Murakami, H.; Nomura, T.; Nakashima, N. Chem. Phys. Lett. 2003, $378,481$.

[55] Gifford, B. J.; Weight, B. M.; Kilina, S. J. Phys. Chem. C 2019, 123, 24807.

[56] Han, J.; Ji, Q.; Qiu, S.; Li, H.; Zhang, S.; Jin, H.; Li, Q. Chem. Commun. 2015, 51, 4712.

[57] Gomulya, W.; Rios, J. M. S.; Derenskyi, V.; Bisri, S. Z.; Jung, S.;
Fritsch, M.; Allard, S.; Scherf, U.; dos Santos, M. C.; Loi, M. A. Carbon 2015, 84, 66.

[58] Chen, F.; Wang, B.; Chen, Y.; Li, L.-J. Nano Lett. 2007, 7, 3013.

[59] Jakubka, F.; Schieß1, S. P.; Martin, S.; Englert, J. M.; Hauke, F.; Hirsch, A.; Zaumseil, J. ACS Macro Lett. 2012, 1, 815.

[60] Rice, N. A.; Subrahmanyam, A. V.; Laengert, S. E.; Adronov, A. J. Polym. Sci., Part A: Polym. Chem. 2015, 53, 2510.

[61] Lei, T.; Chen, X.; Pitner, G.; Wong, H. S.; Bao, Z. J. Am. Chem. Soc. 2016, 138,802 .

[62] Fong, D.; Adronov, A. Macromolecules 2017, 50, 8002.

[63] Hwang, J.-Y.; Nish, A.; Doig, J.; Douven, S.; Chen, C.-W.; Chen, L.-C.; Nicholas, R. J. J. Am. Chem. Soc. 2008, 130, 3543. 